\title{
Increased erythropoietin levels as a biomarker of pancreatic adenocarcinoma: A case report
}

\author{
RAFAY KHAN, QIANG NAI, PING ZHANG, HONGXIU LUO, SHRAMAN SEN, IBRAHIM SIDHOM, \\ TEENA MATHEW, MOHAMMAD ISLAM, SHUVENDU SEN and ABDALLA YOUSIF
}

Department of Internal Medicine, Raritan Bay Medical Center, Perth Amboy, NJ 07733, USA

Received August 5, 2015; Accepted October 20, 2015

DOI: $10.3892 / \mathrm{mco} .2015 .667$

\begin{abstract}
Pancreatic cancer is one of the deadliest cancers commonly diagnosed at an advanced stage. Early diagnosis is crucial for the timely and potentially curative treatment of this highly fatal disease. Although screening tests have improved the survival rate in malignancies such as colon, breast, cervical and prostate cancer, there is currently no effective screening method available for the early detection of pancreatic cancer. As the sensitivity and specificity of existing biomarkers, such as carbohydrate antigen 19-9, for the early detection of pancreatic cancer is low, there is a pressing need for the identification of novel cancer markers. An increase in erythropoietin (EPO) levels has been observed in several cases of pancreatic neoplasms. However, the potential role of EPO as a biomarker of pancreatic cancer or malignant transformation requires further investigation. We herein present a case of increased EPO levels in an adult male patient with stage IV pancreatic cancer.
\end{abstract}

\section{Introduction}

Pancreatic cancer accounts for $\sim 3.0 \%$ of new cancer cases and is the fourth leading cause of cancer-related mortality, accounting for $6.9 \%$ of all cancer deaths worldwide, with an increasing incidence (1). The poor prognosis of pancreatic cancer is mainly attributed to the lack of early diagnostic tools, resulting in limited treatment options, as tumor resectability becomes increasingly limited with advancing cancer stage. Approximately $80 \%$ of pancreatic cancers are not diagnosed until they are locally advanced or have already metastasized, at which point there is no curative therapy, with a median survival of only $8-12$ or 5-8 months, respectively (2). Further

Correspondence to: Dr Rafay Khan, Department of Internal Medicine, Raritan Bay Medical Center, 530 New Brunswick Avenue, Perth Amboy, NJ 07733, USA

Email: Rafay.t.khan@gmail.com

Abbreviations: EPO, erythropoietin

Key words: pancreatic cancer, adenocarcinoma, erythropoietin, malignancy research has been implemented in identifying potential tumor markers or diagnostic tools to help physicians recognize the potential presence of pancreatic cancer, rather than discovering it incidentally on imaging studies at a more advanced stage. Erythropoietin (EPO) has been found to be a potential cancer biomarker. Ectopic EPO production has also been reported in metastatic pancreatic carcinoid tumor (3), pancreatic ductal adenocarcinoma (4) and polycystic pancreatic neoplasms (5).

\section{Case presentation}

A 59-year-old male patient with a past medical history of stage IV pancreatic cancer with metastases to the liver and lung, a history of pulmonary embolism post-inferior vena cava filter placement, intra-abdominal thrombosis, hypercholesterolemia and hypertension, presented to the emergency room with complaints of shortness of breath. The dyspnea had started a few weeks prior, was progressive and was associated with productive cough with yellowish sputum. The patient had no chest pain, fever, chills or palpitations.

The patient had been diagnosed with stage IV pancreatic cancer in August, 2013. A computed tomography (CT) scan of the abdomen was conducted at the time for right-sided abdominal pain, which revealed a focal hypodensity of the body and tail of the pancreas. These findings were consistent with pancreatic carcinoma and metastatic disease was also evident, as there were multiple hepatic lesions. The imaging findings at the time also included splenic, renal, and left portal vein thrombosis. The patient underwent fine-needle aspiration of a liver mass, which was diagnosed as adenocarcinoma; the tumor cells were positive for cytokeratin (CK)7, CK19 and carbohydrate antigen (CA)19-9, and negative for CD56, S100, chromogranin, synaptophysin and CK20. These findings were consistent with a primary pancreatic tumor. The patient underwent 26 cycles of 48-h chemotherapy over the course of 8 months, at which point he developed resistance and the therapy was discontinued for 1 month prior to his admission. The patient was subsequently placed on hospice 2 weeks prior to presentation to the hospital, which was revoked at the family's request due to a new drug (Tarceva) three days later. However, due to the cost of this drug, therapy was put on hold.

On initial physical examination, the patient had a blood pressure of $147 / 54 \mathrm{mmHg}$, a heart rate of $93 \mathrm{bpm}$, a respiratory rate of $14 / \mathrm{min}$, a temperature of $97.5^{\circ} \mathrm{F}$ and a saturation of $92 \%$ 


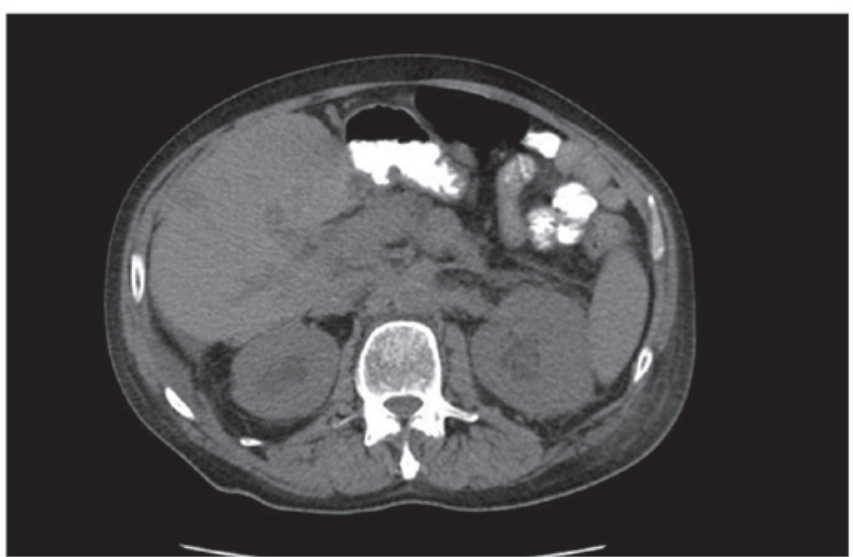

Figure 1. Computed tomography scan of the chest-abdomen-pelvis with findings suggestive of pancreatic neoplasm with hepatic metastases.

on 41 of oxygen. The patient was found to be in moderate distress, with bilateral rhonchi that were more prominent at the bases of the lungs. The extremities exhibited minimal non-pitting edema. The laboratory findings revealed a mild leukocytosis of $11.4 \times 10^{9} / 1$, a hemoglobin concentration of $11.1 \mathrm{~g} / \mathrm{dl}$, a hematocrit of 33.4 and a platelet count of $202,000 / \mathrm{mm}^{3}$. The sodium level was $134 \mathrm{mmol} / 1$, potassium $5.1 \mathrm{mEq} / \mathrm{L}$, chloride $92 \mathrm{mEq} / \mathrm{L}$, bicarbonate $26 \mathrm{mmol} / \mathrm{l}$, blood urea nitrogen $67 \mathrm{mg} / \mathrm{dl}$, and creatinine $8.3 \mathrm{mg} / \mathrm{dl}$. The other initial laboratory findings were non-significant. The chest $\mathrm{X}$-ray revealed a bilateral infiltrate, with bilateral pleural effusions. Due to the presence of anemia and sudden decreases in the hemoglobin concentration, an anemia workup was conducted. The iron level was increased to $193 \mathrm{ng} / \mathrm{ml}$, ferritin to $186 \mathrm{ng} / \mathrm{ml}$, haptoglobin to $86 \mathrm{mg} / \mathrm{dl}$, reticulocyte count to $0.4 \%$, iron-binding capacity to $312 \mu \mathrm{g} / \mathrm{dl}$, whereas the iron saturation was also increased to $62 \%$. The vitamin B12 level was increased to $1,532 \mathrm{ng} / 1$, the folate level was within normal limits and the EPO level was increased to $25.2 \mathrm{mU} / \mathrm{ml}$. On repeat CA19-9 testing, the levels had significantly increased to $2,245 \mathrm{U} / \mathrm{ml}$ from the previous value of $962 \mathrm{U} / \mathrm{ml}$ at the time of initial diagnosis of pancreatic cancer.

The patient was admitted to the intensive care unit due to respiratory distress and acute renal failure; he was initiated on Zosyn and received one dose of vancomycin. Further imaging studies revealed not only findings of liver metastasis, but also metastasis to the lungs, resulting in respiratory distress. Repeat CT imaging of the chest-abdomen-pelvis two days later revealed moderate bilateral hydronephrosis and bilateral perinephric stranding, indicating ureteral obstruction, possibly associated with metastatic disease; however, a stone or obstructing mass could not be excluded at the time (Fig. 1). The lungs exhibited bilateral pulmonary metastatic disease and bilateral pneumonia.

On the following day, the patient had a right nephrostomy tube placed and the renal function began to improve steadily. During his hospital course, however, the patient's condition continued to deteriorate and the patient eventually went into respiratory failure and required intubation. After a discussion with the patient's family regarding his prognosis following failure to wean off the ventilator for 5 days, the family placed a do-not-resuscitate order and the patient was returned to hospice care. The patient was transferred to a long-term care facility where he eventually succumbed to the disease after 1 month.

\section{Discussion}

Unlike the mortality rates of other cancers, such as cancer of the stomach, lungs, colon and prostate, which have been declining over the past 40 years, the rate of pancreatic cancer has remained unchanged or increased in elderly patients, signifying that it is one of the deadliest malignancies (6). Surgically resectable pancreatic cancer has been shown to be associated with an improved prognosis, as it is commonly diagnosed at an early stage. However, an early diagnosis is not always possible, as most symptoms associated with pancreatic cancer are relatively unspecific. Such symptoms include, but are not limited to, abdominal pain, weight loss, new-onset diabetes, jaundice, nausea and vomiting. As it is either incidentally diagnosed or found at a later stage, when the symptoms become progressively worse, only $20 \%$ of the patients with pancreatic cancer are candidates for a potentially curative resection (7).

Pancreatic cancer may develop through several stages of pancreatic intraepithelial neoplastic lesions that may progress from benign to malignant. There have been different gene changes during these phase changes, including K-ras in the early stage, p16 in the intermediate, and p53 in the late stage (8). Although these genetic alterations have been extensively investigated, their diagnostic significance and etiology remain unclear. Exosomes and telomeres are promising markers for early diagnosis; however their clinical utility is still under investigation $(9,10)$.

As mentioned earlier, tumor markers suitable for screening are lacking and the available markers are not recommended for initial testing, even when underlying malignancy is a concern. The markers that have been implemented include carcinoembryonic antigen and CA19-9; however these have relatively insignificant sensitivity and specificity for diagnostic purposes. CA19-9 has been used to monitor the progression of pancreatic cancer but is not typically used as an initial diagnostic tool.

As the characteristics and symptoms of pancreatic cancer are non-specific, identifying sensitive and specific biomarkers is crucial. An incidental finding of elevated EPO levels may suggest an underlying malignancy or may be correlated with pancreatic adenocarcinoma. However, further investigation is required to correlate the elevated levels of EPO to pancreatic cancer or any other malignancy.

As seen with a few other documented case presentations, our patient displayed increased EPO levels as an incidental finding during a routine anemia workup. EPO is a glycoprotein produced by peritublar fibroblasts in the renal cortex and is a regulator of red blood cell formation. The level of EPO is usually low; however, when there is hypoxia or loss of red blood cells, it may increase within a few hours due to increased production by the kidneys. An EPO receptor has been identified in a number of cells and tissues in the body. However, ectopic forms of EPO have been shown to be associated with certain malignancies, which include not only the kidney, but the liver and cerebellum as well. These malignancies include renal cell 
carcinoma, hepatocellular carcinoma, cerebellar hemangioblastoma, ovarian cancer and pheochromocytoma $(5,11)$. EPO receptors have also been reported to occur in tumor cells and, as EPO enhances cell proliferation through cell signaling, an EPO receptor antagonist may be a potential tool for reducing the tumor growth. Recently, an elevated EPO level has been found in pancreatic malignancies, including multicystic neoplasms of the pancreas (5), metastatic pancreatic carcinoid tumor (3) and in pancreatic ductal adenocarcinoma (4). In a previous study, EPO receptors were identified in breast cancer cells, while adjacent normal cells did not display any such receptors (12).

This brings into question whether testing for EPO receptors should also be implemented in the follow-up, or even the initial diagnostic workup of other malignancies, such as pancreatic cancer. Through this case presentation, an association was demonstrated between elevated EPO level and pancreatic adenocarcinoma, suggesting that EPO may be used as an early diagnostic measure or used similarly to CA19-9 to monitor tumor progression and prognosis. This association between EPO and the pancreas remains to be fully elucidated, and further investigation is required to determine its role in tumor growth and its potential use as a cancer biomarker.

\section{References}

1. Howlader N, Noone AM, Krapcho M, Garshell J, Miller D, Altekruse SF, Kosary CL, Yu M, Ruhl J, Tatalovich Z,Mariotto A, Lewis DR, Chen HS, Feuer EJ, Cronin KA (eds): SEER Cancer Statistics Review, 1975-2012, National Cancer Institute. Bethesda, MD, http://seer.cancer.gov/csr/1975_2012/, based on November 2014 SEER data submission, posted to the SEER web site, April 2015.

2. Sultana A, Smith CT, Cunningham D, Starling N, Neoptolemos JP and Ghaneh P: Meta-analysis of chemotherapy for locally advanced and metastatic pancreatic cancer. J Clin Oncol 25: 2607-2615, 2007.
3. Samyn I, Fontaine C, Van Tussenbroek F, Pipeleers-Marichal M and De Grève J: Paraneoplastic syndromes in cancer: Case 1. Polycythemia as a result of ectopic erythropoietin production in metastatic pancreatic carcinoid tumor. J Clin Oncol 22: 2240-2242, 2004.

4. Kawai H, Kojima M, Yokota M, Iguchi H, Wakasugi H, Jimi A and Funakoshi A: Erythropoietin-producing pancreatic ductal adenocarcinoma. Pancrease 21: 427-429, 2000.

5. Nai Q, Regeti K, Arshed S, Hossain MA, Zhang P, Luo H, Singh S, Mathew T, Islam M, Sen S, et al: Elevated erythropoietin and multicystic neoplasm of the pancreas. Case Rep Oncol 8: 148-152, 2015.

6. Kaur S, Baine M, Jain M, Sasson AR and Batra SK: Early diagnosis of pancreatic cancer: Callenges and new developments. Biomark Med 6: 597-612, 2012.

7. Yeo CJ and Cameron JL: Improving results of pancreaticoduodenectomy for pancreatic cancer. World J Surg 23: 907-912, 1999.

8. Maitra A, Kern SE and Hruban RH: Molecular pathogenesis of pancreatic cancer. Best Pract Res Clin Gastroenterol 20: 211-226, 2006.

9. Melo SA, Sugimoto H, O'Connell JT, Kato N, Villanueva A, Vidal A, Qiu L,Vitkin E, Perelman LT, Melo CA, et al: Cancer exosomes perform cell-independent microRNA biogenesis and promote tumorigenesis. Cancer Cell 26: 707-721, 2014.

10. Hou L, Joyce BT, Gao T, Liu L, Zheng Y, Penedo FJ, Liu S, Zhang W, Bergan R, Dai Q, et al: Blood telomere length attrition and cancer development in the normative aging study cohort. EBioMedicine 2: 591-596, 2015.

11. Bunn HF: Erythropoeitin. Cold Spring Harb Perspect Med 3: a011619, 2013.

12. Acs G, Zhang PJ, Rebbeck TR, Acs P and Verma A: Immunhistochemical expression of erythropoietin and erythropoietin receptor in breast carcinoma. Cancer 95: 969-981, 2002. 